\title{
O risco de dietas veganas em idade gestacional e pediátrica: hipóteses, evidências e
}

\section{recomendações}

The risk of vegan diets at gestational and pediatric age: hypotheses, evidence and recommendations

El riesgo de las dietas veganas en la edad gestacional y pediátrica: hipótesis, evidencia y recomendaciones

Recebido: 05/08/2021 | Revisado: 10/08/2021 | Aceito: 11/08/2021 | Publicado: 15/08/2021

Carlos Daniel Silva

ORCID: https://orcid.org/0000-0002-7997-5155

Centro Universitário de Patos de Minas, Brasil E-mail: carlosdaniel8613@gmail.com

Bárbara Queiroz de Figueiredo

ORCID: https://orcid.org/0000-0003-1630-4597

Centro Universitário de Patos de Minas, Brasil

E-mail: barbarafigueiredo@unipam.edu.br

Bárbara Oliveira Vasconcelos Souto

ORCID: https://orcid.org/0000-0003-3399-469X

Centro Universitário de Patos de Minas, Brasil

E-mail: barbarasouto710@hotmail.com

Ana Paula Ferreira Araújo

ORCID: https://orcid.org/0000-0002-6998-7558

Centro Universitário de Patos de Minas, Brasil

E-mail: anapfaraujo@unipam.edu.br

Bruno Faria Coury

ORCID: https://orcid.org/0000-0002-9236-8552

Centro Universitário de Patos de Minas, Brasil

E-mail: brunofaria@unipam.edu.br

Karenn Cristina Neves Bomfim

ORCID: https://orcid.org/0000-0002-8883-4741

Universidade Joé do Rosário Vellano, Brasil

E-mail: kcristinanevesbomfim@gmail.com

Kerolyn Keshyley de Sousa

ORCID: https://orcid.org/0000-0002-4508-8718

Centro Universitário de Brasília, Brasil

E-mail: kerolynks@hotmail.com

Luana Damaceno Miranda

ORCID: https://orcid.org/0000-0003-4013-2414

Centro Universitário de Patos de Minas, Brasil

1 E-mail: uanad.m@hotmail.com

Matheus Gontijo Guimarães Gaia

ORCID: https://orcid.org/0000-0001-9800-8972

Centro Universitário Atenas, Brasil

E-mail: matheusggaia@hotmail.com

Sarah Rabelo Fernandes

ORCID: https://orcid.org/0000-0003-0409-4772

Centro Universitário de Patos de Minas, Brasil

E-mail: sarahrabelo@unipam.edu.br

Rúbia Carla Oliveira

ORCID: https://orcid.org/0000-0002-7162-3497

Centro Universitário de Patos de Minas, Brasil E-mail: rubiacoliveira@unipam.edu.br

\section{Resumo}

Introdução: A alimentação onívora é de suma importância para o desenvolvimento das crianças, principalmente até os nove anos de idade, visto que, é por meio de suplementações presentes em artigos animais, majoritariamente, que desenvolvem capacidades cognitivas, motoras, sensitivas e linguísticas do infante. Assim, uma dieta restritiva pode vir a comprometer o natural desenvolvimento da criança, devido a carência calórica-proteica, sinalizada por meio de fadigas, falta de concentração, falhas na memória, alucinações, icterícias e redução nas capacidades cognitivas, deficiências. Objetivo: Rever a bibliografia atual, a mais relevante e disponível em formato online, sobre os riscos hipotéticos e empíricos, bem como recomendações das dietas vegetarianas em idade gestacional e 
pediátrica. Métodos: Trata-se de uma pesquisa descritiva do tipo revisão integrativa da literatura. Resultados: Crianças que mantém uma dieta restrita ao veganismo, têm maiores riscos de défices fisiológicos. Sob essa perspectiva, deve haver um equilíbrio do aporte de nutrientes, tendo em conta o estado de desenvolvimento da criança, para assegurar um harmonioso crescimento e desenvolvimento. Conclusão: Diante da escolha de inserir uma criança, mesmo nos primeiros anos de vida, numa dieta vegetariana ou vegana, o trabalho do pediatra é respeitar e aconselhar a família, alertando sobre seus riscos. Para isso, a consulta periódica ao pediatra e nutricionista é eminentemente necessária.

Palavras-chave: Pediatria; Veganismo; Desnutrição; Vitamina B12; Ferro.

\begin{abstract}
Introduction: Omnivorous food is of paramount importance for the development of children, especially up to nine years of age, since it is through supplements present in animal articles, mostly, that they develop the infant's cognitive, motor, sensitive and linguistic abilities. Thus, a restrictive diet can compromise the child's natural development, due to caloric-protein deficiency, signaled through fatigue, lack of concentration, memory failure, hallucinations, jaundice and reduced cognitive abilities, deficiencies. Objective: To review the current bibliography, the most relevant and available online, on hypothetical and empirical risks, as well as recommendations for vegetarian diets in gestational and pediatric ages. Methods: This is a descriptive research of the integrative literature review type. Results: Children who maintain a diet restricted to veganism are at greater risk of physiological deficits. From this perspective, there must be a balance in the supply of nutrients, taking into account the child's developmental state, to ensure a harmonious growth and development. Conclusion: When choosing to insert a child, even in the first years of life, on a vegetarian or vegan diet, the pediatrician's job is to respect and advise the family, warning of its risks. For this, periodic consultation with the pediatrician and nutritionist is eminently necessary.
\end{abstract}

Palavras-chave: Pediatrics; Veganism; Malnutrition; B12 vitamin; Ferro.

\begin{abstract}
Resumen
Introducción: La alimentación omnívora es de suma importancia para el desarrollo de los niños, especialmente hasta los nueve años, ya que es a través de los complementos presentes en los artículos animales, en su mayoría, que se desarrollan las habilidades cognitivas, motoras, sensitivas y lingüísticas del infante. Así, una dieta restrictiva puede comprometer el desarrollo natural del niño, por deficiencia calórico-proteica, señalada por fatiga, falta de concentración, fallas de memoria, alucinaciones, ictericia y disminución de las capacidades cognitivas, deficiencias. Objetivo: Revisar la bibliografía actual, la más relevante y disponible en línea, sobre riesgos hipotéticos y empíricos, así como recomendaciones de dietas vegetarianas en edades gestacional y pediátrica. Métodos: Se trata de una investigación descriptiva del tipo revisión integradora de la literatura. Resultados: Los niños que mantienen una dieta restringida al veganismo tienen un mayor riesgo de sufrir déficits fisiológicos. Desde esta perspectiva, debe existir un equilibrio en el aporte de nutrientes, teniendo en cuenta el estado de desarrollo del niño, para asegurar un crecimiento y desarrollo armoniosos. Conclusión: Al optar por insertar a un niño, incluso en los primeros años de vida, en una dieta vegetariana o vegana, el trabajo del pediatra es respetar y asesorar a la familia, advirtiendo de sus riesgos. Para ello es eminentemente necesaria la consulta periódica con el pediatra y nutricionista.
\end{abstract}

Palabras clave: Pediatría; Veganismo; Desnutrición; Vitamina B12; Planchar.

\title{
1. Introdução
}

É fato que o movimento vegano vem crescendo exponencialmente durante os últimos anos, e isso deve-se a diversas questões, tais como a coerção ao sofrimento de animais e a alegação de que a dieta vegetal é rica e suficiente. O vegetarianismo e o veganismo têm sido foco intenso de debate sobre possíveis riscos e benefícios que podem acarretar ao desenvolvimento infantil, principalmente, nas primeiras fases da vida: gravidez, lactação e infância, e caracteriza-se por um padrão alimentar baseado na exclusão da ingesta de carne, subdividindo-se em alguns tipos, conforme o nível de restrição de produtos de origem animal. Essa gama de dietas vegetarianas acaba por dificultar a tarefa de comparar e contrastar os possíveis benefícios à saúde desse tipo de alimentação. Já uma dieta vegana é baseada, exclusivamente, em alimentos vegetais, ou seja, abstém-se de todos os derivados de origem animal, como carne, peixe, ovos, laticínios e mel.

O hominídeo foi capaz de desenvolver sua capacidade motora e de cognição e relações intracromossomiais específicas que resultaram na intelectualidade e inteligência racional, por efeito da dieta rica em vitamina B12, que também é de suma importância para o sangue e para evitar anemia perniciosa. Além disso, por ser hidrossolúvel, ela ajuda a manter o metabolismo do sistema nervoso e das hemácias saudáveis, bem como reduz o risco de danos no DNA e trabalha no controle de níveis de homocisteína, um aminoácido muito associado a doenças cardíacas. Ademais, estudo de Penninx, et al. (2000) 
concluiu que doses periódicas de vitamina B12 diminuem o risco de pessoas desenvolverem depressão, principalmente na puberdade.

Além disso, o cálcio, que é essencial para a formação e mineralização óssea, contração muscular e impulsos nervosos, também tem um papel importante na prevenção de fraturas que são comuns durante a infância. Este mineral também está envolvido nos processos de transporte de nutrientes nas células, na secreção hormonal, na ativação e liberação de enzimas em várias vias de metabolismo, na coagulação sanguínea, na contração muscular, na transmissão de impulsos nervosos e, ainda, na regulação da função muscular cardíaca. No início da puberdade, e com o novo pico no crescimento, também há maior absorção e deposição de cálcio nos ossos. Portanto, cerca de metade do cálcio ósseo presente na idade adulta é acumulado durante a adolescência. Alguns estudos demonstram que o baixo consumo alimentar de cálcio na infância e na adolescência, pode causar déficit de crescimento e de desenvolvimento durante a puberdade, maior probabilidade de desenvolver sobrepeso ou obesidade, aparecimento de doenças ósseas (fraturas com maior frequência e osteoporose na fase adulta e idosa).

Ademais, os ácidos graxos do tipo ômega-3, que estão presentes nos ovos, peixes e algas, apresentam diversas funções na fisiologia humana, particularmente, como componentes das membranas celulares e precursores na síntese de prostaglandinas, leucotrienos e eicosanoides. De acordo com González, et al. (2017), a ingestão desse ácido em idade entre 4 e 12 anos, corrobora na melhora em aspectos cognitivos, como a atenção ao realizar tarefas de brincadeiras ao ar livre, além de atuar na pressão arterial de forma protetora. Além disso, observou-se uma melhora na imunidade nessa faixa etária, diminuindo infecções comuns durante o período, principalmente sintomas de resfriados, bem como na melhoria da compreensão auditiva e na aquisição de vocabulário, diminuição de escores de ansiedade e timidez.

Desse modo, hodiernamente, nota-se que a alimentação onívora é de suma importância para o desenvolvimento das crianças, principalmente até os nove anos de idade, visto que, é por meio de suplementações presentes em artigos animais, majoritariamente, que desenvolvem capacidades cognitivas, motoras, sensitivas e linguísticas do infante. Em elo a isso, depreende-se que, é durante os primeiros anos de vida em que ocorre um colossal desenvolvimento neurológico. Assim, uma dieta restritiva pode vir a comprometer o natural desenvolvimento da criança, devido a carência calórica-proteica, sinalizada por meio de fadigas, falta de concentração, falhas na memória, alucinações, icterícias e redução nas capacidades cognitivas, deficiências.

Infere-se, portanto, que as crianças que mantém uma dieta restrita ao veganismo, têm maiores riscos de défices fisiológicos. Médicos alertam para casos de raquitismo e acidente vascular cerebral (AVC) em crianças que seguem dietas veganas radicais, sem controle e sem suplementos. Sob essa perspectiva, deve haver um equilíbrio do aporte de nutrientes, tendo em conta o estado de desenvolvimento da criança, para assegurar um harmonioso crescimento e desenvolvimento. Desse modo, o objetivo do presente estudo é rever a bibliografia atual, a mais relevante e disponível em formato online, sobre os riscos hipotéticos e empíricos, bem como recomendações das dietas vegetarianas em idade gestacional e pediátrica.

\section{Metodologia}

Trata-se de uma pesquisa descritiva do tipo revisão integrativa da literatura, que buscou responder rever a bibliografia atual, a mais relevante e disponível em formato online, sobre os riscos hipotéticos e empíricos, bem como recomendações das dietas vegetarianas em idade gestacional e pediátrica. A pesquisa foi realizada através do acesso online nas bases de dados National Library of Medicine (PubMed MEDLINE), Scientific Electronic Library Online (Scielo), Cochrane Database of Systematic Reviews (CDSR), Google Scholar, Biblioteca Virtual em Saúde (BVS) e EBSCO Information Services, no mês de julho de 2021. Para a busca das obras foram utilizadas as palavras-chaves presentes nos descritores em Ciências da Saúde (DeCS): em inglês: "pediatrics", "veganism", "malnutrition", "vitamin b12", "iron", em português: "pediatria", "veganismo", "desnutrição", "vitamina b12", "ferro" e em espanhol: "pediatría", "veganismo", "desnutrición", "vitamina b12", "hierro" 
Como critérios de inclusão, foram considerados artigos originais, que abordassem o tema pesquisado e permitissem acesso integral ao conteúdo do estudo, publicados no período de 2001 a 2021, em inglês, português e espanhol. O critério de exclusão foi imposto naqueles trabalhos que não estavam em inglês, português e espanhol que não tinham passado por processo de Peer-View e que não abordassem os riscos hipotéticos e empíricos, bem como recomendações das dietas vegetarianas em idade gestacional e pediátrica. A estratégia de seleção dos artigos seguiu as seguintes etapas: busca nas bases de dados selecionadas; leitura dos títulos de todos os artigos encontrados e exclusão daqueles que não abordavam o assunto; leitura crítica dos resumos dos artigos e leitura na íntegra dos artigos selecionados nas etapas anteriores. Assim, totalizaram-se 24 artigos científicos para a revisão integrativa da literatura, com os descritores apresentados acima. Após esta seleção, filtraram-se por artigos dos últimos vinte anos e por artigos em línguas portuguesa, inglesa e espanhola.

\section{Resultados e Discussão}

A prevalência de dietas restritivas, principalmente vegetarianas e veganas, está aumentando significativamente na Europa e em outros países ocidentais. Em crianças e adolescentes, não apenas o peso e altura, mas também o desenvolvimento neurocognitivo e psicomotor são fortemente influenciados com base nas fontes nutricionais, quantidade e qualidade de sua nutrição. Em estudos feitos principalmente em adultos populações, uma dieta baseada em vegetais mostrou benefícios na redução do risco de doenças crônicas, como como obesidade, diabetes tipo 2, doenças cardiovasculares e alguns tipos de câncer. No entanto, não há evidências claras de que uma dieta vegana iniciada na primeira infância confere uma saúde duradoura beneficiar. Por outro lado, uma dieta vegana pode ser potencialmente crítica para crianças pequenas com riscos de fornecimento inadequado em termos de qualidade de proteína e energia, bem como gordura de cadeia longa ácidos, ferro, zinco, vitamina $\mathrm{D}$, iodo, cálcio e, particularmente, vitamina B12, sendo que deficiências nestes nutrientes podem levar a distúrbios de desenvolvimento graves e às vezes irreversíveis (Müller, 2020).

Qualquer que seja o motivo pelo qual se decida seguir a dieta vegetariana, deve-se conhecer suas vantagens e desvantagens, especialmente, na infância. A maior controvérsia decorre dos possíveis riscos causados pelo déficit de nutrientes, podendo comprometer o desenvolvimento infantil. Proteínas, ácidos graxos, ômega 3, ferro, cálcio, zinco, iodo, vitaminas D e B12, riboflavina e selênio são os nutrientes envolvidos em funções biológicas vitais durante o crescimento e que podem ser insuficientes nessa dieta e, principalmente, na vegana (Cabral, et al. 2020). Durante a idade pediátrica existe maior demanda nutricional inerente ao desenvolvimento da criança. Optar por dietas restritivas pode comprometer o natural desenvolvimento estaturo-ponderal e cognitivo devido à carência proteico-calórica, de vitaminas e de minerais. Este risco é maior nas dietas mais restritivas, não planeadas, e depende de cada indivíduo (Guerra et al., 2012).

A vitamina B12 está presente nas carnes bovinas, suínas, de aves, de peixes, nos ovos, nas vísceras, e em menor quantidade nos leites e derivados, e é ausente nos vegetais, exceto os fortificados. Atua na formação da porção heme da hemoglobina, na maturação dos glóbulos vermelhos e na formação da bainha de mielina no sistema nervoso (Weffort, et al. 2017). Indivíduos lacto-ovovegetarianos, se consomem estes produtos regularmente, em geral recebem quantidades adequadas da vitamina. Porém, vegetarianos estritos são grupo de risco para deficiência e o fato de consumirem quantidades maiores de alimentos ricos em ácido fólico pode mascarar os sintomas hematológicos da deficiência de B12, a anemia megaloblástica. As manifestações neurológicas são: parestesias, hiperreflexia, clônus, irritabilidade, apatia, deterioração mental e demência. A ingestão regular de vitamina B12 em alimentos fortificados deve ser encorajada, e a suplementação é recomendada, especialmente nas lactantes, caso não haja consumo regular destes alimentos (Renda et al., 2009).

De acordo com estudo de Aguirre et al. (2019), filhos de mães deficientes em B12 podem nascer com deficiência ou manifestá-la posteriormente, bem como ingestão adequada e absorção de B12 durante a gravidez e a amamentação são os fatores de proteção mais relevantes do que o estado dos depósitos maternos. Outras condições de risco materno são anemia 
perniciosa, acloridria, dano ileal e desvio gástrico. Embora haja nove relatos em filhos de mães veganos que foram tratados, a prevalência no hospital da pesquisa aumentou rapidamente: 6 casos entre 2006 e 201310 (0,85 casos / ano), 2 entre 2014 e 2016 ( 1 caso / ano) e 7 no período 2016-2018 (3,5 casos / ano). Desse modo, nota-se que a anemia megalobástica, que pode ser corroborada pelo hábito vegano das mães, são raras, mas em número crescente, dada a divulgação do vegetarianismo. Assim, na dieta vegana, não suplementada, as reservas de vitamina B12 cedidas em ambiente intrauterino para o feto são muito escassas, sendo necessária a reposição diária da vitamina B12 para a lactante e para o lactente afetado. Sendo importante ressaltar que as opções nutricionais atuais devem sempre ser acompanhadas por um profissional de saúde experiente (Garcia, et al., 2009).

Além disso, a insuficiência e a deficiência de vitamina D, proveniente de fontes animais e de suma importância para homeostasia do cálcio e metabolismo ósseo, são comuns em lactentes alimentados exclusivamente ao seio, principalmente se nascidos prematuros (quando tiveram menos tempo de acumular depósitos transferidos da mãe pela placenta), filhos de mães que tiveram hipovitaminose D durante a gestação, dieta vegetariana e má absorção intestinal (Alves, et al., 2016).

Outros problemas podem manifestar-se a longo prazo. Exemplo disso são as famílias que optam por uma dieta vegetariana estrita para os seus filhos e que utilizam fórmulas infantis à base de soja para substituir o leite materno. A fórmula de soja, apesar de poder ser usada como substituto do leite de vaca, apresenta elevada concentração de alumínio, fitatos e fitoestrogénios da classe das isoflavonas, e ainda não existem estudos que comprovem os seus efeitos a longo prazo após uma exposição tão precoce. (Yen, et al., 2008). A curto prazo por si só a proteína de soja tem pouco valor nutricional, mas não compromete o desenvolvimento dos lactentes em comparação com os lactentes não vegetarianos. Visto que os efeitos a longo prazo ainda carecem de estudos que comprovem riscos associados, serão exploradas as carências nutricionais associadas ao regime vegetariano e suas variantes (Barranha, 2017).

Em estudo de Dri, et al. (2021), que buscou evidenciar os possíveis atrasos antropométricos em crianças veganas, constatou-se que, além de alguns lactentes cuja mães seguiram período gestacional sob dieta vegana apresentarem menor peso ao nascer quando comparados ao grupo controle, descreve o desenvolvimento puberal em 10 meninos e 10 meninas que seguiram dietas veganas. Após a obtenção de dados por questionário autorreferencial e a idade da menarca por método retrospectivo, em sete crianças o desenvolvimento da genitália ocorreu em idades acima da média da população, o desenvolvimento da mama em 5 meninas ocorreu abaixo da média.

$\mathrm{Na}$ última década tem-se constatado um interesse crescente pelo significado e consequências de estados de hiperhomocisteinemia, que aparenta estar diretamente relacionados com a diminuição de vitamina B12. Desse modo, analisando a história alimentar, a dieta revelou ser um fator preponderante para a ocorrência de um evento trombótico devido aumento dos níveis de homocisteína, sendo que a dieta vegana está associada a défices nutricionais importantes, com desequilíbrios metabólicos que não são inócuos (van Beynum et al., 2005). Segundo relato de caso apresentado por Amaral et al. (2018), elo fato de a mãe fazer uma dieta vegana, as reservas de vitamina B12 cedidas em ambiente intrauterino para o feto foram muito escassas, não tendo sido complementadas no período pós-natal pela introdução de carne na dieta. Assim, a cedência do grupo metilo do folato para a homocisteína através da metionina sintetase sofreu um bloqueio metabólico, pela ausência do cofator necessário à reação, a vitamina B12, levando ao estado de hiperhomocisteinemia no lactente e na mãe, com aumento do risco pró-trombótico.

No entanto, estudo de Schürmann et al. (2017) concluiu que o desenvolvimento físico de infantes vegetarianos e onívoros era semelhante e dentro da faixa normal, e em ambos os grupos, a ingestão de energia e proteína atendeu a referência de valores, enquanto a ingestão de ferro foi de $60-70 \%$ de acordo com os padrões de referência. Em vegetarianos, a ingestão de vitamina $\mathrm{C}$ excedeu a referência valores de referência $(70 \mathrm{mg} / \mathrm{d})$. A análise de amostra de sangue mostrou uma maior prevalência de deficiência de ferro em vegetarianos do que em onívoros (ferritina sérica média: 9,61 vs 36,1 $\mu \mathrm{g} / \mathrm{L}$ ), e os 
níveis de lipídios no sangue foram encontrados dentro da faixa normal em ambos os grupos. Em 50 vegetarianos britânicos com idades entre 7-11 anos, medidas de peso corporal, altura corporal, circunferência do braço e dobras cutâneas mostraram uma semelhança no desenvolvimento físico em relação ao grupo controle onívoro, enquanto a altura final calculada era maior (em $0,47 \mathrm{~cm}$ ) em vegetarianos.

A consciência desses nutrientes potencialmente críticos permite aos pais que planejam uma dieta vegana para eles próprios e seus filhos para tomarem decisões informadas em seus. Escolha de alimentos e suplementos. Sob essa perspectiva, nota-se que as proteínas vegetais têm uma composição de aminoácidos menos diversificada do que aqueles de animais, por isso é importante estar ciente de fontes específicas de diferentes proteínas vegetais e para aumentar a ingestão, a fim de evitar a falta de aminoácidos essenciais. Isso é especialmente importante porque as crianças precisam de aproximadamente 30\% maior ingestão por até 2 anos, 20-30\% até 6 anos e 15-20\% para crianças mais velhas (Messina, et al., 2001). Ademais, fontes de comida vegana geralmente têm um maior teor de fibra e podem, portanto, levar a um déficit na ingestão de energia, especialmente em bebês e crianças pequenas devido à saciedade e plenitude prematuras. A este respeito, deve-se prestar atenção a uma densidade de energia adequada da comida. No caso do bebê alimentado por veganos, a amamentação é geralmente recomendada por pelo menos os primeiros 6 meses de vida, como com qualquer recém-nascido. Se a amamentação não for possível, uma fórmula infantil à base de soja pode ser usada, sendo segura em termos de crescimento, metabólico, endocrinológico, reprodutivo, e funções neurológicas (Vandenplas, et al., 2014).

A falta dietética de ácidos graxos poliinsaturados essenciais, como ácidos graxos ômega-3, pode corroborar o mau desenvolvimento neurológico, como lentidão no desenvolvimento da retina. Assim, o ácido docosahexaenóico e o ácido eicosapentaenóico, que estão presentes principalmente em produtos de origem animal, devem ser suficientemente supridos em crianças veganas, por exemplo, com alfa-linolênico, o precursor desses ácidos. A inclusão de óleo de linhaça e nozes também podem suprir a escassez de ácidos graxos ômega-3 (Craddock, et al., 2017).

Além de seu papel na síntese de hemoglobina, o ferro é importante para a mielina, haja vista a ação de bainhas de mielinas nervosas e para a síntese de neurotransmissores. A demanda por de ferro na primeira infância e a adolescência aumentam em comparação com os adultos, e a bio-disponibilidade de ferro heme $(\mathrm{Fe} 2+)$, que é normalmente encontrado na carne, é melhor do que a do ferro não heme ( $\mathrm{Fe} 3+)$, sendo a absorção deste último, dependente dos alimentos consumidos ao mesmo tempo. Portanto, deve haver cuidado para que os inibidores da absorção de ferro, como fitatos de leguminosas e compostos de cálcio do leite não sejam tomados simultaneamente com importantes fontes de alimentos ferrosos (van Winckel, et al., 2011).

O zinco também é inibido em sua absorção pelo ácido fítico. Como um elemento essencial e cofator para muitas enzimas, os sintomas clínicos da deficiência de zinco são diversos, como cicatrização de feridas prejudicada, fragilidade das unhas, queda de cabelos, susceptibilidade à infecção, bem como diarreia crônica e crescimento prejudicado (Roohani, et al., 2013). Na ausência de ferro de origem animal, deve-se prestar atenção às fontes de alimentos ricos em zinco, como cereais, produtos fermentados de soja e nozes. Para deficiência clínica de zinco, suplemento adicional é necessário (5 mg de $\mathrm{Zn} /$ dia para crianças de 6 a 36 meses, $10 \mathrm{mg}$ de Zn / dia para crianças mais velhas) (Brown, et al., 2004).

Embora a vitamina D seja encontrada em vários alimentos de origem animal, como laticínios e peixes gordurosos, as necessidades desta vitamina são principalmente cobertas pela produção endógena por pele irradiada com raios ultravioleta. O uso de produtos fortificados ou suplementação com 400-600 UI / dia é recomendada para todos os bebês e crianças. A falta de leite e produtos lácteos na dieta também reduz a suprimento de cálcio, especialmente quando o bebê é desmamado do leite materno. Aliado a isso, na criança em crescimento, uma suficiente ingestão de cálcio é importante para a obtenção de uma densidade óssea ideal (pico de massa óssea), e é uma oportunidade única para reduzir o risco de fraturas e osteoporose mais 
tarde na vida. Vegetais verdes com baixo teor de oxalato, como brócolis, repolho chinês e a couve são boas fontes de cálcio, bem como bebidas purificadas, cereais e água mineral rica em cálcio completam as opções alimentares (Baroni, et al., 2018).

A vitamina B12 (cobalamina) em uma forma biologicamente ativa não está disponível em fontes não-animais, e uma dieta vegana deve, portanto, ser regularmente suplementada. A vitamina B12 é essencial para o corpo realizar muitas funções essenciais, como eritropoiese, síntese de mielina, homeostase dos axônios e até mesmo metabolismo mitocondrial para obtenção de energia (Honzik, et al., 2010). Sua deficiência, que também pode ocorrer na criança amamentada por uma mãe com deficiência de vitamina B12, pode levar a doenças neurológicas graves, às vezes irreversíveis dano psicológico e atraso no desenvolvimento (Dror, et al., 2008). A dose diária de cobalamina, que deve ser administrada por via oral como um suplemento em lactência e infância, ainda não está estabelecida, no entanto, sugestões recomendam doses diárias de 5 ug para bebês e crianças pequenas. Alguns produtos comerciais disponíveis usam vias de administração, como spray nasal ou creme dental suplementado com vitamina B12 que ainda é pouco estudada para absorção adequada na infância (Agnoli, et al., 2017).

\section{Conclusão}

As crianças, por crescerem e desenvolverem rapidamente, são consideradas um grupo de risco por não apresentarem um sistema imunológico totalmente competente, podendo ser expostas à vários tipos de doenças, e quando expostas a um quadro de desnutrição, principalmente no primeiro ano de vida pode acarretar em diversos problemas de saúde pública, como infecções de repetição, problemas no crescimento e desenvolvimento psicomotor, comprometimento na vida escolar, inúmeros problemas na vida adulta, hematológicos como a anemia nas suas várias formas carenciais. Como conclusão provisória, a maioria estudos apresentados não mostraram efeito prejudicial fixa de dietas vegetarianas em crianças, no entanto, os riscos aumentados de dietas vegetarianas para a saúde foram particularmente relatados para status de ferro.

Conclui-se, portanto, que diante da escolha de inserir uma criança, mesmo nos primeiros anos de vida, numa dieta vegetariana ou vegana, o trabalho do pediatra é respeitar e aconselhar a família, alertando sobre seus riscos. Em geral, essa dieta não altera o desenvolvimento infantil, desde que muito bem planejada e diversificada. Para isso, a consulta periódica ao pediatra e nutricionista é eminentemente necessária. Ademais, por fazer parte da formação neurológica da criança, a vitamina B12 deve ser monitorada e suplementada via oral, a fim de evitar danos irreversíveis, além do controle e, se preciso, da suplementação de ferro, zinco e cálcio. Por fim, é importante destacar que, em qualquer padrão alimentar, a dieta deve ser baseada em produtos de qualidade, priorizando frutas e vegetais; sem excesso de açúcares, sal e aditivos sintéticos e com o menor processamento possível, visto que alimentos ultraprocessados de origem vegetal ainda são tão desaconselháveis quanto aqueles ultraprocessados de origem animal. Desse modo, estudos prospectivos totalmente conduzidos em bebês, crianças e adolescentes em dietas veganas ou vegetarianas, a fim de comparação com grupos de controle onívoros são urgentemente necessários, haja vista a relevância do tema como saúde pública.

\section{Referências}

Agnoli, C., Baroni, L., \& Bertini, I. (2017). Position paper on vegetarian diets from the working group of the Italian Society of Human Nutrition. Nutr Metab Cardiovasc Dis. 27 (1), 1037-1052.

Aguirre, J. A., Donato, M. L., Buscio, Ceballos, V., Armeno, M., Aizpurúa, L. \& Arpí, L. (2019). Comprometimento neurológico sério devido à vitamina B12 e deficiência em bebês de mães veganas e vegetarianas Arch Argent Pediatr., 117 (4), 420-424.

Alves, C. A. D. \& Cargnin, K. R. N. (2016). Hipovitaminose D em pediatria: recomendações para o diagnóstico, tratamento e prevenção. Departamento Científico de Endocrinologia - Sociedade Brasileira de Pediatria, 1-11.

Amaral, J., Ezequiel, M. \& Luis, C. (2018). Acidente Vascular Cerebral Isquémico num Lactente Filho de Mãe Vegana. Acta Pediatr Port, 49 (1), 66-70.

Baroni, L., Goggi, S. \& Battaglino R. (2018). Vegan nutrition for mothers and children: practical tools for healthcare providers. Nutrients. 11 (5), 1-5.

Barranha, S. G. W. (2017). Alimentação Vegetariana em Idade Pediátrica: Riscos, benefícios e recomendações. Mestrado Integrado em Medicina. Porto. 1-37. 
Brown, K. H., Rivera, J. A., \& Bhutta, Z. (2004). International Zinc Nutrition Consultative Group (IZiNCG) technical document \#1. Assessment of the risk of zinc deficiency in populations and options for its control. Food Nutr Bull. 25 (2), 99-203.

Cabral, A. A., Ventura, T. B., Bonazzio, A. B., Torres, V. M., Mourão, B. A., Santana, D. \& Silva, C. P. (2020). Implicações Nutricionais da Alimentação Vegetariana e Vegana no Desenvolvimento Infantil: uma Revisão Narrativa. Residência Pediátrica, 332 (1), 1-17.

Craddock, J. C., Neale, E. P., Probst, Y. C. \& Peoples, G. E. (2017). Algal supplementation of vegetarian eating patterns improves plasma and serum docosahexaenoicacid concentrations and omega-3 indices: a systematic literature review. J Hum Nutr Diet. 30 (1), $693-699$.

Dri, J., Mejía, R. R., Santos, E. G. \& Pino, M. (2021). Crecimiento en niños y en hijos de madres que adhieren a dietas vegetarianas: revisión de la literatura. Arch Argent Pediatr. 119(4), 77-106. http://dx.doi.org/10.5546/aap.2021.S77

Dror, D. K. \& Allen, L. H. (2008). Effect of vitamin B12 deficiency on neurodevelopment in infants: current knowledge and possible mechanisms. Nutr Rev. $66(1), 250-255$.

Garcia, C. P., Cardoso, A. L., Carneiro, J. D. A. \& Macedo, A. C. L. (2009). Deficiência de vitamina B12 em lactente alimentado com leite materno. Rev Bras Nutr Clin. 25 (3), 264-266.

González, F. E. \& Báez, R. V. (2017). Importância do ômega 3 na nutrição infantil. Revista Paulista de Pediatria, 35 (1), 3-4. https://doi.org/10.1590/1984$0462 / ; 2017 ; 35 ; 1 ; 00018$

Guerra, A., Rêgo, C., Silva, D., Ferreira, G.C., Mansilha, H. \& Antunes H. (2012). Alimentação e nutrição do lactente. Acta Pediátrica Port. 43 (5), 17-40.

Honzik, T., Adamovicova, M. \& Smolka, V. (2010). Clinical presentation and metabolic consequences in 40 breastfed infants with nutritional vitamin B12 deficiency - what have we learned? Eur J Paediatr Neurol. 14 (1), 488-495.

Messina, V. \& Mangels, A. R. (2001). Considerations in planning vegan diets: children. J Am Diet Assoc. 101 (1), $661-669$.

Müller, P. Vegan Diet in Young Children. Nestlé Nutr Inst Workshop Ser. Basel. 93(1), 103-110.

Penninx, B. W. J. H., Guralnij, J. M., Ferrucci, L., Fried, L. P., Allen, R. H. \& Stabler, S. P. (2000). Deficiência e depressão de vitamina B 12 em mulheres idosas com deficiência física: evidências epidemiológicas do estudo de saúde e envelhecimento da mulher. The American Journal of Psychiatry, 157 (1), 715 721.

Renda, M. \& Fischer, P. (2009). Dietas vegetarianas em crianças e adolescentes. Pediatr Rev. 30 (1), 1-8.

Roohani, N., Hurrell, R., Kelishadi, R. \& Schulin, R. (2013). Zinc and its importance for human health: An integrative review. J Res Med Sci. 18 (1), 144157.

Schürmann, S., Kersting, M. \& Alexy, U. (2017). Vegetarian diets in children: a systematic review. Eur J Nutr, 1-21.

van Beynum, I. M., den Heijer, M., Thomas, C. M. G., Afman, L., Emmerzaal, D. O. \& Blom, H. J. (2005). Total homocysteine and its predictors in Dutch children. Am J Clin Nutr. 81 (5), 1110-1116.

van Winckel, M., Vande, V. S, de Bruyne R. \& van Biervliet, S. (2011). Clinical practice: vegetarian infant and child nutrition. Eur J Pediatr. 170 (1), 14891494.

Vandenplas, Y., Castrellon, P. G. \& Rivas R. (2014). Safety of soya-based infant formulas in children. Br J Nutr. 111 (1), $1340-1360$.

Weffort, V. \& Lamounier, J. A. (2017). Nutrição em Pediatria: da infância a adolescência, (2a ed.), Editora Manole.

Yen, C. E., Yen, C. H., Huang, M. C., Cheng, C. H. \& Huang, Y. C. (2008). Dietary intake and nutritional status of vegetarian and omnivorous preschool children and their parents in Taiwan. Nutr Res. 28 (7), 430 - 436. 